\title{
DYNAMIC LIGHT SCATTERING INVESTIGATION OF PNIPAM-co-MAA MICROGEL SOLUTION
}

\author{
TOMASZ ŚLIWA ${ }^{\mathrm{a}, \mathrm{b}}$, MACIEJ JARZĘBSKI ${ }^{\mathrm{a}, \mathrm{b}}$ \\ aAdam Mickiewicz University, Faculty of Physics, Molecular Biophysics Division, Umultowska 85, 61-614 Poznań, Poland \\ ${ }^{\mathrm{b}}$ NanoBioMedical Centre, Adam Mickiewicz University, Umultowska 85, 61-614 Poznań, Poland.
}

Received February 27, 2014; accepted March 20, 2014; published online March 25, 2014

\begin{abstract}
The paper presents results of a study of the effect of the environment temperature and $\mathrm{pH}$ on the size of particles based on poly N (isopropylacrylamide) chain. The tested substance was the copolymer PNIPAM-co-MAA. The particle size measurements were performed by dynamic light scattering. It was found that the copolymer tested reacts specifically to temperature increase by shrinking more than two times. Important for stabilization of the structure are the chemical groups -COOH present in methacrylic acid that undergoes dissociation. Gradual increase in temperature results in a decrease in the dissociation constant, in binding of protons and thus causes shrinkage of the entire particle. It was also shown that PNIPAM-co-MAA in high concentrations undergoes crystallization.
\end{abstract}

\section{INTRODUCTION}

Nanotechnology is one of the fastest developing branches of science. Nanoparticles are defined as structures whose diameter ranges between 1 and $100 \mathrm{~nm}$ (Ravichandran, 2009). In practice, molecules with diameters close to $1000 \mathrm{~nm}$ are also treated as nanoparticles. One of the most interesting present or future applications of nanoparticles is in medicine as drug carriers for targeted drug delivery or as contrast agents (Sattler 2010).

Many classifications of nanoparticles for medical applications have been proposed. Faraji and Wipf have divided nanostructures for drug delivery into: inorganic particles, polymers, solid lipids, liposomes, nanocrystals and nanotubes (Faraji \& Wipf, 2009). Polymers belong to the most popular materials, whose properties, such as particle size or addition of external or internal of reactive centers can be easily controlled.

Multi-stimuli responsive polymer nanoparticles have been recently widely studied as a promising material in medical application. The unique properties of poly $(\mathrm{N}-$ isopropylacrylamide) (PNIPAM) based polymers and copolymers predestined them as innovative drug carriers for drug delivery systems (Chen et al., 2012) diagnostic substance carriers and or as biosensors (i.e. nanotermometer) (Chen \& Chen, 2011).

PNIPAM-based polymers undergo changes in conformation in response to external stimuli such changes in $\mathrm{pH}$, temperature, ionic strength, salt concentration, electric and magnetic field, light or ultrasounds (Meid et al 2012, Schmaljohann 2006). The materials which react specifically to changes in their environment are also called "smart materials" (Karg, Hellweg 2009). PNIPAM-base polymers exhibit the ability to change their properties from the hydrophilic, in low temperatures, to hydrophobic, in high temperatures. The critical temperature of the phase transition (LCST) is in the range of $32-34^{\circ} \mathrm{C}$ (Chen, Jiang, \& Sun, 2013). The phenomenon of particle shrinkage is based on the dipole interaction between polymer chains, which can be used in specific immobilization of some antibodies (Zhou, Veron, Elaissari, Delair \& Pichot, 2004). Also the hydrogen bonds play an important role in the shrinkage behavior (Kratz, Hellweg \& Eimer, 2000), that may be caused by interaction of $-\mathrm{COOH}$ groups coming from the copolymeric part of the chain. The consequence of changes in these interactions is the binding of water by a hydrophilic molecule that causes its swelling below LCST and above LCST a sharp contraction of the molecule takes place and the water, previously bounded inside, is released to the environment. This process may be corrupted by addition of ethanol to the solution, because ethanol particles adhere to the polymer chain and substitute the water bonded inside the particle (Biswas et al, 2012). The effect of change in the size and release of the inner substance can be potentially used in controlled drug delivery systems in which PNIPAM would act as a carrier of an active substance or drug would release it in a targeted place (Eeckman et al, 2004, Margaret A. Phillips et al, 2010). The particles 
may also take the complex form like core-shell type with a magnetic core and PNIPAM chain shell (Zhang \& Wang, 2009). This composition allows transportation of the particle by magnetic field to the place of drug release and heats it up for a more effective PNIPAM collapse.

\section{MATERIALS AND METHODS}

\section{Polymer synthesis}

Polymer synthesis was made following the procedure described in (Ito et al, 1999). In brief P(NiPAM-coMAA) was synthesized from NiPAM and methacrylic acid (MAA). The cross-linker was BIS (N,N-methylenebisacrylamide). The polymerization process was initiated by KPS (potassium persulfate). For the fluorescence tests the sample was dyed by rhodamine B. PNiPAM microgel solution was centrifuged, then freeze-dried.

\section{Hydrodynamic radius}

In this study, we measured the hydrodynamic radius of the particles by dynamic light scattering (DLS). The measuring system was based on a goniometer (ALV, Germany) with a correlator ALV/5000E and the avalanche photodiode (SPCM-AQR). The laser system used in this experiment was a $\mathrm{HeNe} 633 \mathrm{~nm}$.

The principles of the DLS method are given in detail in (Berne \& Pecora, 1976). In general the scattered light intensity fluctuates because of the Brownian motion of the particles. The intensity autocorrelation function is given by Eq. (1):

$$
g^{2}(\tau)=B\left[1+\beta\left|g^{1}(\tau)\right|^{2}\right],
$$

where $B$ is the baseline, $\beta$ is the coherence factor. The $g^{l}(\tau)$ stands for the 1-st order (field) autocorrelation function and for monodisperse solution is given by Eq. (2):

$$
g^{1}(\tau)=\exp (\Gamma \tau)
$$

Where $\Gamma$ is proportional to the square of scattering angle $q$, Eq. (3):

$$
\Gamma=D q^{2},
$$

where $D$ is the diffusion coefficient obtained from the experiment. For a mixture of a few components in solution the cumulant expansion of autocorrelation function might be calculated according to Eq. (4):

$$
\ln g^{1}(\tau)=\ln A+\Gamma_{1} \tau+\frac{1}{2 !} \Gamma_{2} \tau^{2}+\frac{1}{3 !} \Gamma_{3} \tau^{3},
$$

This equation is used for the fitting procedure. On the basis of the constants from the cummulant expression, the polydispersity index (pdi) can be calculated from Eq. (5):

$$
p d i=\frac{\Gamma_{2}}{\Gamma_{1}^{2}},
$$

Hydrodynamic radius is obtained indirectly from the diffusion coefficient given by the Stokes-Einstein Eq. (6):

$$
D=\frac{k_{B} T}{6 \pi \eta R_{h}},
$$

where $k_{B}$ is the Boltzmann constant and $\eta$ - the solvent viscosity, $T$ - temperature, $R_{h}$ - hydrodynamic radius.

For the tests PNIPAM-co-MAA was diluted with deionized water to a concentration of $0.02 \mathrm{mg} / \mathrm{ml}$ samples. The solutions were stabilized by buffers (Chempur) at $\mathrm{pH} 2-10$. All tests, DLS and particle tracking were made in the temperature range $20-50^{\circ} \mathrm{C}$ at every 2 degree.

\section{Temperature and $\mathrm{pH}$ dependence}

The tests were made using a standard $\mathrm{pH}$ electrode and a resistive heater. The PNIPAM-co-MAA solution was prepared by dissolving dry polymer in Millipore water without buffer. The final concentration was $0.5 \mathrm{mg} / \mathrm{mL}$. The investigation was carried in a closed test-tube with an embedded $\mathrm{pH}$ electrode. The solution was heated and measured at every $0.2^{\circ} \mathrm{C}$. The $\mathrm{pH}$ measurements were corrected for the effect of temperature changes.

\section{Structural investigations}

The structure of fluorescent PNIPAM particles was studied using confocal microscopy by a Zeiss Laser Scanning Microscope LSM780. The images were taken with a microscope water immersion objective ZEISS CApochromat 40x/1.2 W Corr M27. For the measurements, the chamber coverglass (8 WELL from LABTEK) and filled up slide channels ( $1 \mu$-Slide from Ibidi) were used.

\section{RESULTS AND DISCUSSION}

PNIPAM-co-MAA copolymer was selected for the test because it contains additional - $\mathrm{COOH}$ groups with respect to those in the native PNIPAM, and these groups 
play an important role in the contraction of the molecule caused by $\mathrm{pH}$ and temperature changes. The choice of $\mathrm{pH}$ values was dictated by the $p K a$ of the methacrylic acid that is 4.25 (Tiwari et al 2012). Below the $p K a$ the polymer chains are neutral and carboxylic groups are not dissociated. Above the $p K a$ they became negatively charged, dissociated to COO- and protons and highly hydrated because of the increase in osmotic pressure inside the particle (Himstedt, Marshall \& Wickramasinghe, 2011) that may inhibit the particle collapse.

The DLS results presented in Figs 1-4 illustrate the apparent changes in particles size above the critical temperature. For $p K a$ below the critical value the initial particle size at $20^{\circ} \mathrm{C}$ is around $450 \mathrm{~nm}$ from DLS and it slightly decreases until reaching LCST. At the critical temperature a rapid particle contraction is observed. This behavior might be employed in systems of controlled drug delivery.

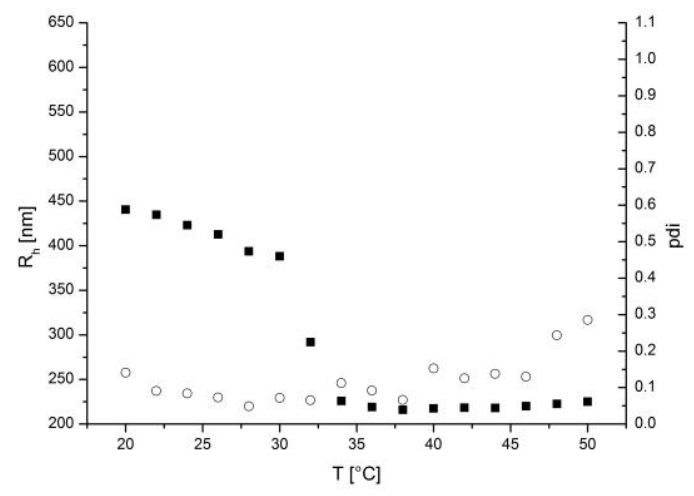

Fig. 1. Hydrodynamic radius (black squares) and polydispersity (white dots) index versus temperature changes at $\mathrm{pH} 2$.

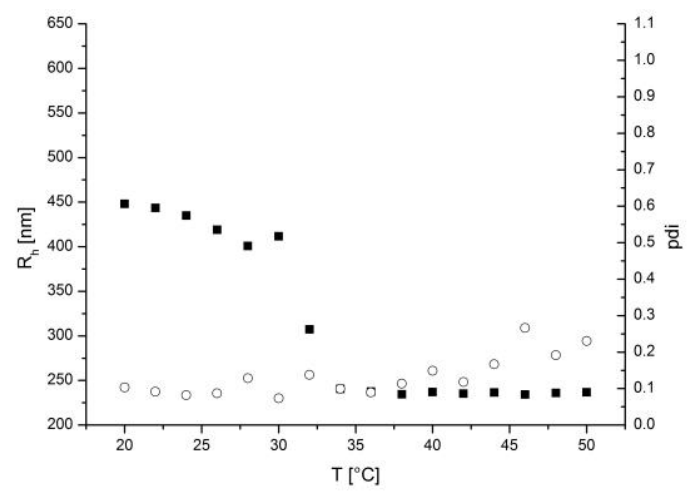

Fig. 2. Hydrodynamic radius (black squares) and polydispersity (white dots) index versus temperature changes at $\mathrm{pH} 3$.

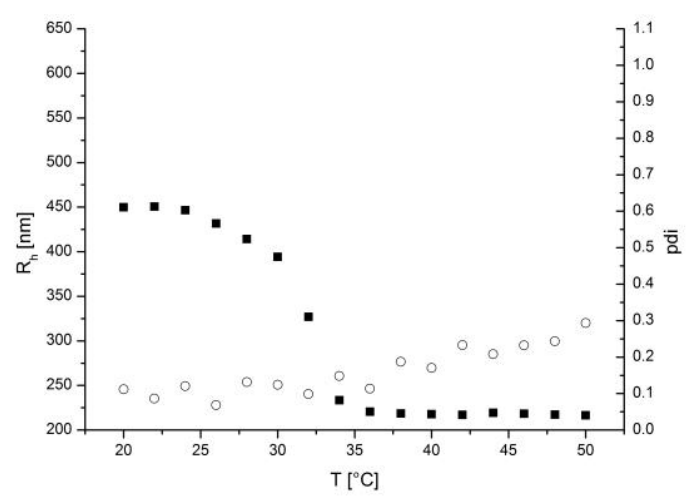

Fig. 3. Hydrodynamic radius (black squares) and polydispersity (white dots) index versus temperature changes at $\mathrm{pH} 4$.

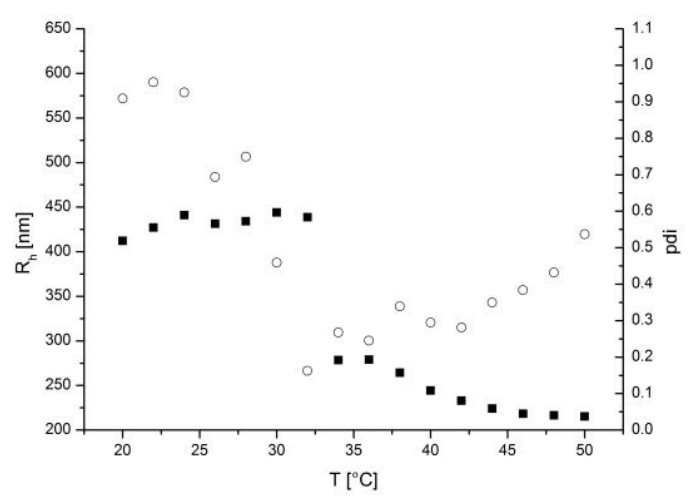

Fig. 4. Hydrodynamic radius (black squares) and polydispersity (white dots) index versus temperature changes at $\mathrm{pH} 5$.

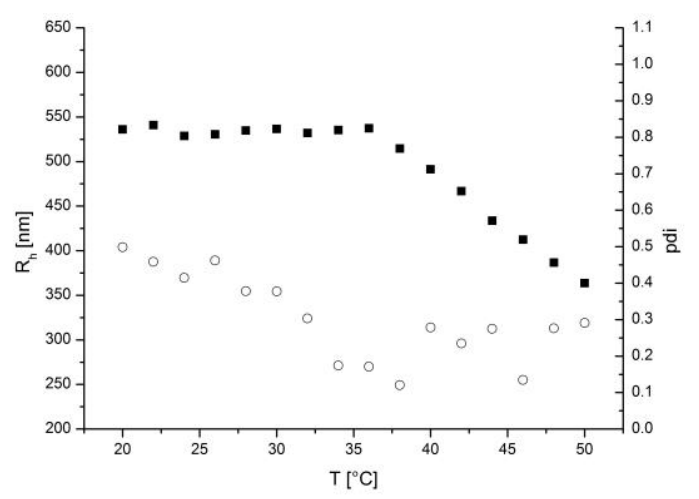

Fig. 5. Hydrodynamic radius (black squares) and polydispersity (white dots) index versus temperature changes at $\mathrm{pH} 6$. 


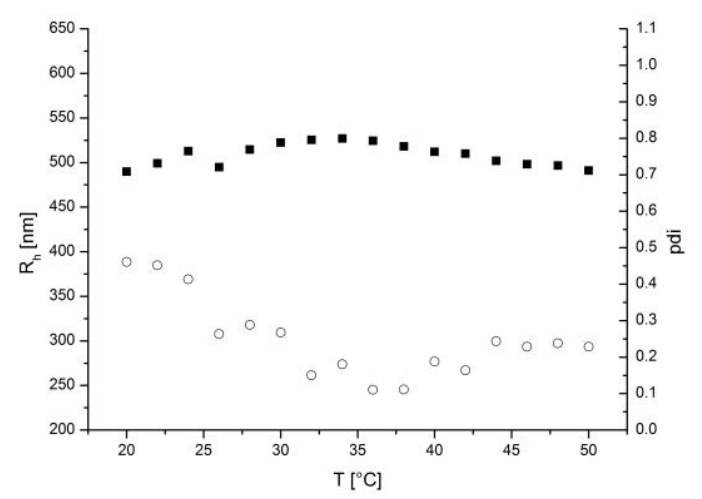

Fig. 6. Hydrodynamic radius (black squares) and polydispersity (white dots) index versus temperature changes at $\mathrm{pH} 7$.

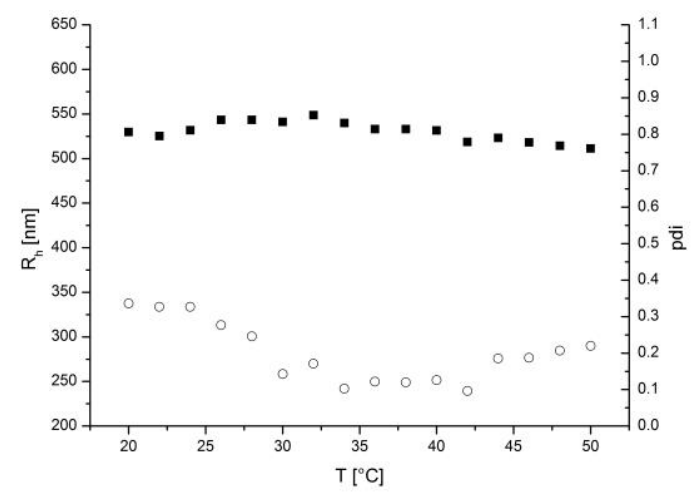

Fig. 7. Hydrodynamic radius (black squares) and polydispersity (white dots) index versus temperature changes at $\mathrm{pH} 8$.

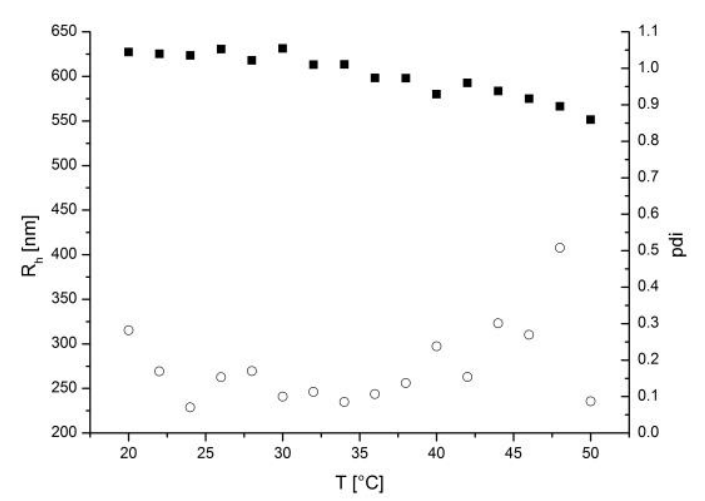

Fig. 8. Hydrodynamic radius (black squares) and polydispersity (white dots) index versus temperature changes at $\mathrm{pH} 9$.

At $\mathrm{pH}$ higher than 5 (Figs 5-8), the average initial particle size is bigger than at $\mathrm{pH}$ lower than 5 and the LCST contraction is unobserved.

At $\mathrm{pH}$ lower than $\mathrm{pKa}$, the pdi is above 0.3 which implies that the particles are quite monodisperse. At $\mathrm{pH} 5$, pdi undergoes unexpected changes that may be interpreted as related to transition state of the molecule.

Above the critical temperature in stabilized environment changes in particle size were observed. This observation has prompted us to make an experiment under the change in $\mathrm{pH}$ in a solution without a buffer. Because change in particle size caused by heating involves modification in hydrophilic properties as a result of changes in the degree of dissociation of chemical groups in the solution, the change in $\mathrm{pH}$ in non-stabilized solution can be expected as a result of temperature changes.

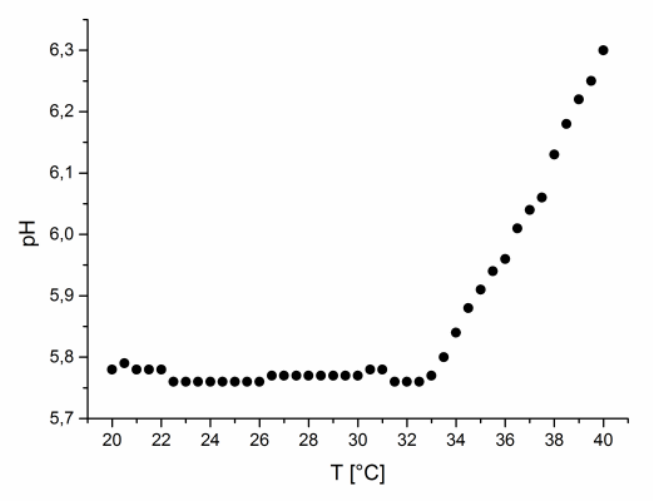

Fig. 9. Temperature dependence of $\mathrm{pH}$

According to the results presented in Fig. 9, below LCST the $\mathrm{pH}$ changes are not observed. When temperature exceeds LCST a marked increase in $\mathrm{pH}$ is noticed that indicates attachment of protons to the dissociated $-\mathrm{COO}^{-}$groups.

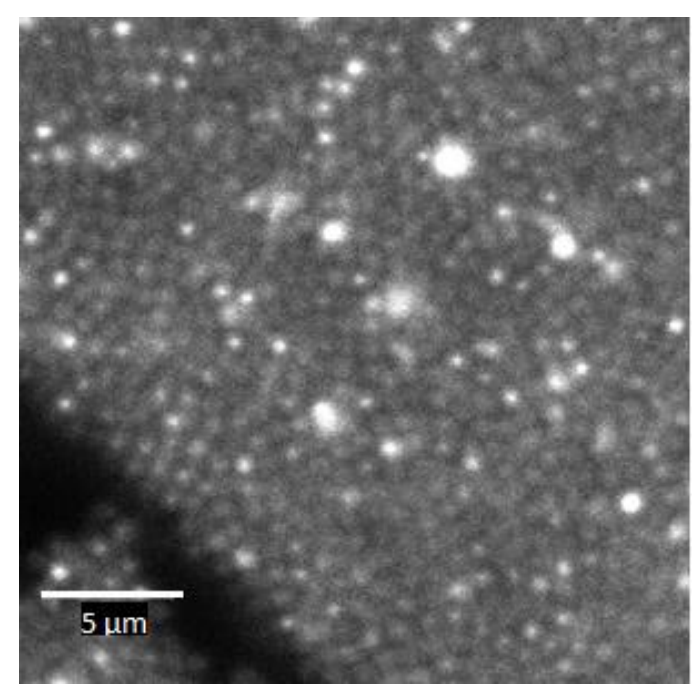

Fig. 10. LSM scan, PNIPAM-co-MAA dyed with rhodamine B. 
PNIPAM-co-MAA particles were labeled with rhodamine $\mathrm{B}$ dye during the synthesis to make them detectable using confocal scanning fluorescence microscopy. The following pictures show the morphology of concentrated solution in water mixture. It can be seen that the particles are characterized by the ability to aggregate at high concentrations, Fig. 10.

\section{CONCLUSION}

The experiments have shown that the copolymer of PNIPAM and methacrylic acid exhibit volume phase transition accompanied with shrinkage in response to temperature change at acidic $\mathrm{pH}$. The observed changes in the particle size are approximately 2-times. Above the $p K a$ of acrylic acid the size of PNIPAM-co-MAA particle is bigger and almost constant on the sample heating.

\section{ACKNOWLEDGMENTS}

Authors would like to thank Dzina Kleshchanok from SABIC Europe, Science and Technology Centre, Geleen for the synthesis of the sample, Adam Patkowski and Jacek Gapinski for help in analysis of the obtained results.

Tomasz Śliwa thanks the NanoBioMedical Centre in Poznań, Adam Mickiewicz University and Faculty of Physics where Ph.D. thesis is carried under the project co-financed by European Union, programme Human Capital National Cohesion Strategy funded by European Social Fund.

Maciej Jarzębski wishes to thank the Foundation for Polish Science and NanoBioMedical Centre in Poznań Adam Mickiewicz University, Faculty of Physics that the work was supported by the International $\mathrm{PhD}$ Projects Programme of Fundation for Polish Science operated within the Innovative Economy Operational Programme (IE OP) 2007-2013 within European Regional Development Fund. Financial support from National Centre for Research and Development under research grant "Nanomaterials and their application to biomedicine" contract number PBS1/A9/13/2012, is gratefully acknowledged.

\section{REFERENCES}

Berne B.J., Pecora R., (1976) Dynamic Light Scattering: with Applications to Chemistry, Biology, and physics, Wiley, New York.

Biswas C.S., Patel VK., Vishwakarma N.K., Mishra A.K., Ray B. (2012). Synthesis and Characterization of Porous Poly(Nisopropylacrylamide) Hydrogels Prepared in Ethanol-Water Mixtures. Journal Of Applied Polymer Science, 121, 2422-
2429.

Chen C. Y. and Chen C. T. (2011) A PNIPAM-Based Fluorescent Nanothermometer with Ratiometric Readout Chem. Commun., 47, pp. 994-996.

Chen S., Jiang X., Sun L. (2013). Effects of end groups on the thermal response of poly(N-isopropylacrylamide) microgels. Journal of Applied Polymer Science, 130, 1164-1171.

Chen S., Zhong H., Gu B., Wang Y., Li X., Cheng Z., Zhang L. \& Yao C., (2012) Thermosensitive phase behavior and drug release of in situ $\mathrm{N}$-isopropylacrylamide copolymer Materials Science \& Engineering C-Materials For Biological Applications, 32, 8, pp. 2199-2204.

Eeckman F, Moës A.J., Amighi K. (2004) Synthesis and characterization of thermosensitive copolymers for oral controlled drug delivery. European Polymer Journal, 40, 873-881.

Faraji A., Wipf P. (2009) Nanoparticles in cellular drug delivery. Bioorganic \& Medicinal Chemistry, 17, 2950-2962.

Himstedt H.H., Marshall K.M., Wickramasinghe S.R. (2011). $\mathrm{pH}$-responsive nanofiltration membranes by surface modification. Journal of Membrane Science, 366, 373-381.

Ito S., Ogawa K., Suzuki H., Wang B., Yoshida R., Kokufuta E. (1999) Preparation of Thermosensitive Submicrometer Gel Particles with Anionic and Cationic Charges. Langmuir, 15, 4289-4294.

Karg M., Hellweg T. (2009). New "smart" poly(NIPAM) microgels and nanoparticle microgel hybrids: Properties and advances in characterization. Current Opinion in Colloid \& Interface Science, 14, 438-450.

Kratz K., Hellweg T, EimeraW. (2000). Influence of charge density on the swelling of colloidal poly( $\mathrm{N}$-isopropylacrylamide-co-acrylic acid) microgels. Colloids and Surfaces A: Physicochemical and Engineering Aspect, 170, 137-149.

Meid J., Dierkes F., Cui J., Messing R., Crosby A. J., Schmidt A. \& Richtering W. (2012). Mechanical properties of temperature sensitive microgel/polyacrylamide composite hydrogels - from soft to hard fillers, Soft Matter, 8, pp. 42544263.

Phillips M.A., Gran L.M., Peppas N.A. (2010) Targeted nanodelivery of drugs and diagnostics, nanotoday 5, 143-159.

Ravichandran R. (2009) Nanotechnology-Based Drug Delivery Systems, Nanobiotechnol., 5, 17-33.

Sattler K.D. (2011). Handbook of Nanophysics: Nanomedicine and Nanorobotics. Handbook of Nanophysic, CRC Press.

Schmaljohann D. (2009). Thermo- and pH-responsive polymers in drug delivery. Advanced Drug Delivery Reviews 58, $1655-1670$.

Tiwari A., Mishra A.K., Kobayashi H., Turner A.P.F., (2012). Intelligent Nanomaterials, Willey.

Zhang F., Wang Ch.Ch, (2009). Preparation of P(NIPAM-coAA) Microcontainers Surface-Anchored with Magnetic Nanoparticle. Langmuir, 25, 8255-8262.

Zhou G., Veron L., Elaissari A., Delair T., Pichot Ch. (2004), A new route for the preparation of cyano-containing poly $(\mathrm{N}-$ isopropylacrylamide) microgel latex for specific immobilization of antibodies. Polymer International, 56, 603-608. 